\title{
Potential public health benefits of HIV testing occurring at home in Australia
}

among 10000

untested gay

and bisexual

men ... use of

a single self-

test ... would

detect about

586 infections

that would

otherwise go

undetected

University of New

South Wales and

St Vincent's Centre

for Applied Medical

Research (UNSW-AMR)

HIV Home Testing

Assessment Group

Sydney, NSW.

Rguy@

kirby.unsw.edu.au

doi: 10.5694/mjal4.01210 n Australia between 1999 and 2013 the annual number of HIV diagnoses rose by over $70 \%$, from 724 to 1236 , and gay and bisexual men (GBM) accounted for $70 \%$ of new cases. ${ }^{1} \mathrm{HIV}$ testing is recognised in the Seventh National HIV Strategy as a key public health strategy. ${ }^{2}$ Increased HIV testing leads to earlier detection of HIV infections, which allows people who are diagnosed to reduce the risk of transmission to others by modifying their sexual practices. ${ }^{3}$ Being diagnosed with HIV also allows people to decide whether they wish to initiate treatment that suppresses viral replication and thereby reduces infectiousness. ${ }^{4}$ Mathematical modelling has predicted that substantial increases in HIV testing can reduce $\mathrm{HIV}$ incidence in the community. ${ }^{5}$

Despite GBM having access to laboratory HIV testing through clinical services, HIV testing rates remain less than ideal. Less than a quarter of high-risk GBM undergo testing at the recommended frequency (3-6 monthly). ${ }^{6}$ Surveys show the proportion of GBM who have never had a test for HIV is $14 \%-26 \%, 7,8$ and in 2013 there were about 3700 people undiagnosed and living with HIV in Australia. ${ }^{1}$ GBM consistently report structural barriers to HIV testing, ${ }^{9,10}$ and $61 \%-67 \%$ report they would undergo testing more frequently if home testing were available. ${ }^{11,12}$

In an effort to increase testing rates in Australia, legislation regulating the availability of in vitro devices was recently modified to allow the Therapeutic Goods Administration to consider applications for licensing of HIV self-tests. Policies have also been changed to support HIV self-testing in other countries, including the United States, United Kingdom, France and Kenya. In the US, the oral fluid OraQuick In-Home HIV Test (OraSure) has been approved by the US Food and Drug Administration (FDA) and is commercially available. Studies in untrained users show the sensitivity of the OraQuick self-test to be $91.67 \%$, but the specificity is nearly $100 \%$, ${ }^{13}$ which means very few false-reactive results occur.

Despite the lower sensitivity of the OraQuick self-test compared with laboratory tests, the approval by the US FDA was based on public health grounds. A mathematical model was developed which assumed that 2.8 million people in the US (seropositivity of $1.6 \%$ ) would use the self-test in its first year of use. Based on the known sensitivity of the self-test, this would yield 45000 positive test results and 3800 false-negative results. The model predicted that, by uncovering this large number of previously undiagnosed infections, the self-test might avert more than 4000 new HIV transmissions during the first year. ${ }^{14}$

Australia's HIV epidemic is quite different to that in the US, with a higher proportion of new infections among GBM. ${ }^{1}$ Our health system also differs, with more widely

\section{Summary}

- In many countries, including Australia, policies have recently changed to support HIV self-testing. The decision has created much debate about the public health benefits of the strategy versus the risks.

- Self-testing for HIV was approved in the US on the basis that it would facilitate greater HIV testing uptake, despite having a lower sensitivity than laboratory HIV immunoassays.

- We calculated the frequency of self-testing that would be required among Australian gay and bisexual men at high-risk for there to be a public health benefit (detection of HIV infections that would have otherwise remained undiagnosed).

- At a population level, if access to HIV self-testing led to men supplementing their usual sexual health check-ups (involving a laboratory HIV immunoassay) with one or more self-tests at home, or self-tests led to untested gay and bisexual men having an HIV test for the first time, there would be a public health benefit.

- If men replaced their average of one laboratory HIV immunoassay per year with self-testing at home, then three self-tests would be needed to counteract the lower sensitivity of the self-test (so zero infections would be missed). If four self-tests were undertaken then additional infections would be detected (ie, there would be a public health benefit).

- Additional public health benefits include a reduction in the period of undiagnosed infection, which is known to be a period of relatively high infectiousness.

available highly sensitive fourth-generation laboratory HIV immunoassays (IAs) and easily accessible inexpensive primary health care. Therefore, it is important to confirm that there would be public health benefits of HIV self-testing before introducing it in Australia. We assessed the potential benefit to public health of HIV self-testing in Australia, defined by the number of HIV infections detected that otherwise would have remained undiagnosed.

\section{Details of our analysis}

We prepared a series of calculations to assess the chance of detecting HIV among Australian GBM at high risk of infection if the OraQuick self-test were used, compared with a laboratory fourth-generation IA. We also assessed what level of HIV testing frequency is required for the number of new HIV infections detected by HIV selftests to offset any infections missed due to the lower sensitivity of the self-test. We applied these calculations to a range of testing scenarios. 


\section{Population studied}

Our analysis focused on men who reported higher behavioural risk, among whom HIV incidence levels are greatest. ${ }^{15}$ Such men also report a greater willingness to use HIV self-tests. ${ }^{11}$ High risk was defined as reporting more than ten sexual partners in the past 6 months, and/ or any unprotected anal intercourse with casual partners, and/or unprotected sex with an HIV-positive partner. For this high-risk population, we applied an HIV incidence of 2.4 per 100 person-years overall and 6.4 per 100 personyears in untested men (Box 1$).^{15}$

\section{Our assumptions regarding test performance}

Based on data from the OraSure clinical trial conducted with participants who had not been trained in how to use the test ${ }^{13}$ we assumed that the self-test, which detects only antibodies in oral secretions, has an overall sensitivity of $91.67 \%$. We also assumed that the window period was $25-35$ days (median 30 days). ${ }^{18}$ We assumed that the fourth-generation IA, which detects p24 antigen (ie, part of the virus) as well as antibody in blood, has a sensitivity of $99.94 \%{ }^{19}$ and a window period of $15-20$ days (median 17.5 days) (Box 2). ${ }^{18}$

\section{Calculating the chance of HIV detection}

We calculated the proportion of infections that would remain undiagnosed if the self-test replaced a single fourth-generation IA laboratory test at an individual level.

We then calculated the chance of HIV detection via test frequencies of a single yearly test, two or more tests in a year, every 1-2 years, and greater than 2 years ago (Box 3). The chance of HIV detection for both the self-test and fourth-generation IA was also assessed (Box 3). We assumed that test sensitivity is the probability of detecting a true positive and that an infection would occur on average at the midpoint between the last true-negative test and the next HIV test.

\section{Calculating the proportion of infections that would go undetected if the OraQuick HIV self- test replaced fourth-generation IA}

Based on the usual testing frequency among high-risk GBM, we calculated the proportion of infections that would go undetected per year if self-tests replaced IA tests. We calculated the weighted average across the proportions of men who test a given number of times per year, $n$, and the probability of not detecting an established infection, using the following equations:

Probability(missing an infection with IA $\mid$ average of one test per year $)=(1-0.9994)^{1}$

Probability(missing an infection with self-test $\mid$ average of $X$ tests per year $)=(1-0.9167)^{X}$

(where " $\mid$ " indicates conditional probability; ie, the probability of event $A$ given that event $B$ occurred).

\section{Parameters for calculations}

Category

Estimate

HIV incidence

High-risk GBM overall*

2.4 per 100 person-years

GBM who have never undergone testing ${ }^{\dagger}$

6.4 per 100 person-years

No. of HIV tests in past 12 months among high-risk GBM ${ }^{\ddagger}$

2

$3-4$

$25 \%$

$32 \%$

$22 \%$

$4 \%$

$\geqslant 5$

$9.5 \%$

High-risk $\mathrm{GBM}^{\ddagger}$ tested every $1-2$ years

$7.0 \%$

GBM = gay and bisexual men. * Summary of HIV incidence estimates in high-risk GBM attending clinical settings; $\geqslant 6$ male sexual partners (2.0 per 100 person-years); inconsistent condom use ( 2.4 per 100 person-years); any sexually transmitted infection diagnosis in past 2 years ( 2.3 per 100 person-years); HIV-positive regular partner (2.7 per 100 person-years). ${ }^{15} \dagger$ Based on HIV incidence in GBM attending clinical services who reported not having a previous HIV test. ${ }^{15}$ ¥High-risk GBM reporting more than ten partners in the past 6 months, and/or unprotected anal intercourse with casual partner, and/or unprotected sex with an HIV-positive partner ${ }^{16,17}$

\section{Test performance estimates}

\begin{tabular}{lcc} 
& \multicolumn{2}{c}{ Estimate } \\
\cline { 2 - 3 } Assay & \multicolumn{1}{c}{ Sensitivity overall } & Window period \\
\hline OraSure OraQuick self-test & $91.67 \%(95 \% \mathrm{Cl}, 84.24-96.33) * 13$ & $25-35$ days $^{18}$ \\
$\begin{array}{l}\text { Fourth-generation } \\
\text { immunoassay }\end{array}$ & $99.94 \%(95 \% \mathrm{Cl}, 99.79-99.99)^{19}$ & $15-20$ days $^{18}$ \\
\hline $\begin{array}{l}\text { * Based on } 106 \text { new infections and eight false-negative results (one person underwent } \\
\text { seroconversion and was excluded). }\end{array}$
\end{tabular}

3 HIV testing frequency and chance of detection

\begin{tabular}{|c|c|c|c|c|c|c|}
\hline \multirow{3}{*}{ Assay } & \multicolumn{6}{|c|}{ Chance of detection* } \\
\hline & \multirow{2}{*}{$\begin{array}{l}\text { Tested >2 } \\
\text { years ago }\end{array}$} & \multirow{2}{*}{$\begin{array}{c}\text { Tested every } \\
1-2 \text { years }\end{array}$} & \multicolumn{4}{|c|}{ Testing frequency in past 12 months } \\
\hline & & & 1 & 2 & $3-4$ & $\geqslant 5$ \\
\hline $\begin{array}{l}\text { Fourth-generation } \\
\text { immunoassay }\end{array}$ & $99.94 \%$ & $99.94 \%$ & $99.94 \%$ & $99.97 \%$ & $99.98 \%$ & $99.99 \%$ \\
\hline $\begin{array}{l}\text { OraSure OraQuick } \\
\text { self-test }\end{array}$ & $91.67 \%$ & $91.67 \%$ & $91.67 \%$ & $95.49 \%$ & $96.97 \%$ & $98.49 \%$ \\
\hline \multicolumn{7}{|c|}{$\begin{array}{l}\text { *For the purpose of calculation, we assumed the test sensitivity is the probability of detecting a true } \\
\text { positive and assuming that an infection would occur on average at the midpoint between the last } \\
\text { true negative test and the next HIV test. For the purpose of calculations, we assumed three tests } \\
\text { were conducted for the category of } 3-4 \text { tests in the last } 12 \text { months, and six tests were conducted for } \\
\text { the category of } \geqslant 5 \text { tests in the last } 12 \text { months. }\end{array}$} \\
\hline
\end{tabular}

Then, to determine what level of HIV testing frequency is required for the number of new HIV infections detected by HIV self-tests to offset any infections missed by the lower sensitivity of the self-test, we used this calculation:

$X=1 * \log (1-0.9994) / \log (1-0.9167)$

We assumed that high-risk GBM had on average one test per year, based on clinical data that showed an average testing frequency of around 1.5 tests per year among men who presented to a clinic for testing ${ }^{20}$ but, as $27 \%$ of men do not attend a clinic for testing in a year, we reduced it to one test per year on average. 


\section{Outcomes of our calculations}

The net benefit of replacing fourth-generation laboratory IA tests with HIV self-tests depends on the frequency of testing in the target population.

If a group of high-risk GBM were going to receive a single fourth-generation IA test, but instead undertook a selftest, then about $8 \%$ of the infections that would have been diagnosed by the IA test would remain undetected due to the lower sensitivity of the self-test.

If self-testing increased across all GBM at high risk so that men had three self-tests per year on average, compared with one conventional fourth-generation IA, then zero infections would be missed. If four self-tests occurred, additional infections would be detected (ie, meeting our definition of public health benefit).

Additional infections would also be detected for any extra self-test which was supplementary to usual fourthgeneration laboratory IA testing. Self-testing could have a large benefit among men who would otherwise remain untested. For example, among 10000 untested GBM, with an incidence of 6.4 per 100 person-years, ${ }^{15} 640$ new infections would occur. Use of a single self-test by these men would detect about 586 infections that would otherwise go undetected.

\section{Discussion}

Results of our calculations show that HIV self-testing would have a public health benefit if access to the self-test led to Australian GBM at high risk of infection supplementing their conventional testing with self-tests. If selftesting resulted in untested GBM having an HIV test for the first time, there would also be a public health benefit (even a test with lower sensitivity is better than no test). A good uptake of self-testing seems plausible, considering the interest expressed in surveys. ${ }^{11,12}$ The uptake of selftests as supplemental testing or by previously untested men would reduce the average period of undiagnosed infection. The approximately $9 \%$ of undiagnosed HIV infections among Australian GBM disproportionately account for a third of new HIV infections. ${ }^{21}$ Mathematical modelling suggests that reducing the time between infection and diagnosis can lead to reductions in population incidence. $^{22}$

If the availability of self-tests resulted in GBM replacing their conventional laboratory test with self-tests, then men would need to undertake three or more self-tests a year to counteract the lower sensitivity of the self-test. Conducting three self-tests a year is consistent with clinical guidelines, but there would need to be systems to facilitate this, such as online ordering and subsidised tests from pharmacies via repeat prescription. If replacement occurred but only two self-tests were undertaken, then HIV infections would be missed. This suggests that if self-testing is introduced, men who are already linked with care would need to be encouraged to continue their annual sexual health check-ups. Recent qualitative research found that most GBM would supplement but not replace conventional blood testing with self-tests, because they valued screening for other sexually transmitted infections and the professional expertise and support provided at health services. ${ }^{23}$

Other considerations for introducing self-tests include cost. GBM in Australia report they would be unwilling to pay more than $\$ 30$ for self-tests, preferably less than $\$ 20,{ }^{24}$ however, in the US a self-test currently costs around US $\$ 40$. This cost still compares favourably to clinic visits, where there are often clinic fees, patient travel costs, clinical staff salaries, infrastructure and pathology costs. Lower rates of linkage to care, and psychological distress after a receiving a reactive self-test result have been raised as potential risks. However, in the US, the OraSure Unobserved Use Study found most people (96\%) identified as HIV-positive reported they intended to seek medical care. ${ }^{25}$ Self-tests kits should have clear instructions about seeking medical care and available support lines.

There are some points to consider when interpreting our results. First, the calculations conducted were simple but provide some examples of the likely benefit of high-risk GBM having access to self-tests. We have provided a rough estimate of the level of change required in practice to offset reductions in sensitivity if replacement were to occur. We realise that it is likely that not all high-risk GBM would use HIV self-tests. Surveys show that about $61 \%-67 \%$ of GBM would undergo testing more frequently if HIV self-tests were available. ${ }^{11,12}$ Our calculations are relevant to the subgroup of GBM who would consider using HIV self-tests.

In October 2013, the first Australian randomised controlled trial of OraQuick oral self-tests in high-risk GBM commenced (called FORTH), which includes two target groups of high-risk GBM; those who test infrequently (last test $>2$ years ago) and test more frequently (past 2 years). ${ }^{26}$ FORTH will provide more accurate information on the actual HIV testing frequency that can be achieved with HIV self-testing and the extent of supplementing versus replacement of conventional tests. Mathematical modelling will be conducted using these estimates.

In conclusion, these calculations suggest that HIV selftesting should be made available to Australian GBM, provided it leads to increased testing among: GBM who have not undergone testing before; men supplementing their conventional testing with home testing; or men replacing their usual conventional testing frequency with four self-tests a year. HIV infections have increased by over $70 \%$ in the past 15 years, ${ }^{1}$ and innovative methods are needed to increase testing and treatment to sufficient levels in order to control the HIV epidemic.

Acknowledgements: Full author details are available in the Appendix.

Competing interests: No relevant disclosures.

Provenance: Not commissioned; externally peer reviewed.

References are available online at www.mja.com.au. 
1 The Kirby Institute. HIV, viral hepatitis and sexually transmitted infections in Australia: annual surveillance report, 2014. Sydney: The Kirby Institute for Infection and Immunity in Society, University of New South Wales, 2014.

2 Australian Government Department of Health. Seventh National HIV Strategy: 2014-2017. Canberra: Commonwealth of Australia, 2014.

3 Marks G, Crepaz N, Senterfitt JW, Janssen RS. Meta-analysis of high-risk sexual behavior in persons aware and unaware they are infected with HIV in the United States: implications for HIV prevention programs. J Acquir Immune Defic Syndr 2005; 39: 446-453.

4 Cohen MS, Chen YQ, McCauley M, et al; HPTN 052 Study Team. Prevention of HIV-1 infection with early antiretroviral therapy. NEngl J Med 2011; 365: 493-505.

5 Jansson J, Kerr CC, Wilson DP. Predicting the population impact of increased HIV testing and treatment in Australia. Sex Health 2014; 11: 146-154.

6 Guy R, Goller JL, Spelman T, et al. Does the frequency of HIV and STI testing among men who have sex with men in primary care adhere with Australian guidelines? Sex Transm Infect 2010; 86: 371-376.

7 Holt M, Rawstorne P, Wilkinson J, et al. HIV testing, gay community involvement and internet use: social and behavioural correlates of HIV testing among Australian men who have sex with men. AIDS Behav 2012; 16: 13-22.

8 Zablotska I, Holt M, de Wit J, et al. Gay men who are not getting tested for HIV. AIDS Behav 2012; 16: 1887-1894.

9 MacKellar DA, Hou SI, Whalen CC, et al; WHBS Study Group. Reasons for not HIV testing, testing intentions, and potential use of an over-the-counter rapid HIV test in an internet sample of men who have sex with men who have never tested for HIV. Sex Transm Dis 2011; 38: 419-428.

10 Prestage G, Brown G, Keen P. Barriers to HIV testing among Australian gay men. Sex Health 2012; 9: 453-458.

11 Bavinton BR, Brown G, Hurley M, et al. Which gay men would increase their frequency of HIV testing with home self-testing? AIDS Behav 2013; 17: 2084-2092.

12 Chen M, Bilardi J, Lee D, et al. Australian men who have sex with men prefer rapid oral HIV testing over conventional blood testing for HIV. Int J STD AIDS 2010; 21: 428-430.

13 United States Food and Drug Administration. OraQuick In-Home HIV Test: summary of safety and effectiveness. FDA, 2012. http://www.fda.gov/downloads/ BiologicsBloodVaccines/BloodBloodProducts/ ApprovedProducts/PremarketApprovalsPMAs/UCM312534. pdf (accessed May 2015).

14 Myers JE, El-Sadr WM, Zerbe A, Branson BM. Rapid HIV selftesting: long in coming but opportunities beckon. AIDS 2013; 27: 1687-1695.
15 Guy RJ, Spelman T, Stoove M, et al. Risk factors for HIV seroconversion in men who have sex with men in Victoria, Australia: results from a sentinel surveillance system. Sex Health 2011; 8: 319-329.

16 Hull P, Mao L, Kao S, et al. Gay Community Periodic Survey: Sydney 2013. Sydney: National Centre in HIV Social Research, University of New South Wales, 2013. https://csrh.arts.unsw. edu.au/media/CSRHFile/GCPS_Sydney_2013_Report.pdf (accessed Apr 2015).

17 Lee E, Mao L, McKenzie T, et al. Gay Community Periodic Survey: Melbourne 2013. Sydney: National Centre in HIV Social Research, University of New South Wales, 2013. https://csrh. arts.unsw.edu.au/media/CSRHFile/GCPS_Melbourne_2013_ Report.pdf (accessed Apr 2015).

18 Branson BM, Stekler JD. Detection of acute HIV infection: we can't close the window. J Infect Dis 2012; 205: 521-524.

19 Chavez P, Wesolowski L, Patel P, et al. Evaluation of the performance of the Abbott ARCHITECT HIV Ag/Ab Combo Assay. J Clin Virol 2011; 52 Suppl 1: S51-S55.

20 Read TRH, Hocking JS, Bradshaw CS, et al. Provision of rapid HIV tests within a health service and frequency of HIV testing among men who have sex with men: randomised controlled trial. BMJ 2013; 347: f5086.

21 Wilson DP, Hoare A, Regan DG, Law MG. Importance of promoting HIV testing for preventing secondary transmissions: modelling the Australian HIV epidemic among men who have sex with men. Sex Health 2009; 6: 19-33.

22 Jansson J, Kerr CC, Wilson DP. Predicting the population impact of increased HIV testing and treatment in Australia. Sex Health 2014; 11: 146-154.

23 Bilardi J, Walker S, Read T, et al. Gay and bisexual men's views on rapid self-testing for HIV. AIDS Behav 2013; 17: 2093-2099.

24 Bradley J, Prestege G, Bavinton B, et al. The TAXI-KAB study: thinking about exposure to infection - knowledge, attitudes and beliefs. Sydney: The Kirby Institute, University of New South Wales, 2012.

25 OraSure Technologies. Final advisory committee briefing materials: available for public release. OraQuick In-Home HIV Test. Bethlehem, PA: OraSure Technologies, 2012. http://www.fda.gov/downloads/ AdvisoryCommittees/CommitteesMeetingMaterials/ BloodVaccinesandOtherBiologics/ BloodProductsAdvisoryCommittee/UCM303652.pdf (accessed May 2015).

26 Kirby Institute, University of New South Wales. Go Forth [trial site]. About the Forth Study. http://www.nchecrsurveys.unsw. edu.au/forth (accessed May 2015) 